\title{
Granulomatosis with polyangiitis: Special focus on suggestive imaging findings in head and neck involvement
}

Elif Bulut*, $[\mathrm{MD}]$

ORCID:0000-0003-0305-2239
* Corresponding Author: Hacettepe University Faculty of Medicine, Department of Radiology

Sihhiye Ankara

Tel: +90 3123051188 +905335735432

Fax: +903123112145

E-mail: drelifbulut@yahoo.com

\section{e) ABSTRACT}

Objective: Granulomatosis with polyangiitis (GPA) is a rare autoimmune disease with frequent upper respiratory tract involvement. Early diagnosis is crucial for early initiation of effective treatment, which improves outcome. Imaging plays an important role for confirming clinically suspected cases. The aim of our study is to reveal the frequencies of imaging findings previously defined as suggestive for GPA (i.e. osseous erosions or destructions, neoosteogenesis, subglottic stenosis) and to investigate frequency and imaging characteristics of previously under-recognized nasopharyngeal involvement in GPA.

Materials and Methods: We retrospectively examined paranasal, nasopharynx/neck, temporal CTs $(n=51)$ and MRIs $(n=14)$ of 48 patients with GPA. The evaluated imaging findings include mucosal ulcerations in nasal cavity, sinonasal and temporal opacifications/inflammatory signal changes, presence and locations of osseous erosions and neoosteogenesis, periantral soft tissue infiltration, septal perforation, presence and characteristics of nasopharyngeal involvement, and subglottic stenosis. Descriptive statistics were presented as mean $\pm \mathrm{SD}$; categorical variables were presented as count and percentage.

Results: Forty patients (87\%) had sinonasal and fourteen (29\%) patients had tympanic cavity \pm mastoid opacifications. Three patients $(23 \%)$ had signal and diffusion characteristics suggesting a sinonasal granulomatous inflammation. Mucosal ulcerations were detected in 32\% of patients. Osseous erosions/destruction (40\%) was common in disease onset and frequently affected mid-line sinonasal structures. Neoosteogenesis of paranasal sinuses (21\%) was more common in follow-up studies and invariably affected maxillary sinuses. Septal perforation (6\%), periantral infiltration (4\%) and subglottic stenosis $(10 \%)$ were relatively infrequent findings. Nasopharyngeal involvement (23\%) was not infrequent; predominantly presented with wall thickening, ulcerations, low T2-weigted signal changes and restricted diffusion in MRI. Conclusion: Sinonasal osseous erosion/destruction is a relatively common and highly suggestive finding especially when co-existent with nasal mucosal ulcerations, neoosteogenesis and nasopharyngeal thickening in a suspected case. Nasopharyngeal involvement is not infrequent and imaging could overlap with other entities such as nasopharyngeal cancer or lymphoma.

Keywords: Granulomatosis with polyangiitis, head and neck, computed tomography, magnetic resonance imaging, nasopharynx

\section{INTRODUCTION}

Granulomatosis with polyangiitis (GPA), formerly known as Wegener's granulomatosis is a rare antineutrophil cytoplasmic antibody (ANCA)associated autoimmune disease. It is characterized by necrotizing granulomatous inflammation and necrotizing vasculitis of small to medium vessels
[1]. It is more frequent between 45 and 60 years of age, affects women and men with similar frequencies [2]. GPA characteristically involves upper respiratory tract, lungs and kidneys; although any organ can be affected. Ear, nose and throat (ENT) involvement is frequent and present in $70 \%$ to $100 \%$ 
of cases at diagnosis [2, 3]. In current clinical practice, the diagnosis of GPA is usually based on elevated c-ANCA levels and histopathologic confirmation. However, up to $\% 50$ of cases with limited GPA have no detectable ANCA $[2,4]$. It could also be difficult to find histopathologic evidence of GPA in some cases. Imaging plays an important role for confirming clinically suspected diagnosis of GPA in such serologically and histopathologically inconclusive patients. The CT imaging characteristics of sinonasal involvement are well-described and involve mucosal thickening, osseous erosions or destructions and neoosteogenesis [5]. Mucosal thickening is a non-specific finding and could be detected in patients with sinusitis of varied etiology. Nodular thickening may favor the diagnosis of GPA [6]. Bony changes on the other hand are suggestive of GPA when clinical suspicion is high [5, 7]. Subglottic stenosis is known as the most frequent airway manifestation and characterized by circumferential thickening, irregularity and ulceration [5]. The imaging findings of nasopharyngeal and temporal involvement are less well recognized compared to sinonasal and laryngeal involvement. The limited radiologic data comes from single cases or small case series of GPA involvement in nasopharynx mimicking nasopharyngeal cancer [8-10].

The aim of our study was to reveal the relative frequencies of more specific imaging findings (i.e., osseous erosions or destructions, neoosteogenesis, subglottic stenosis) and to investigate the prevalence and imaging characteristics of previously under-recognized nasopharyngeal involvement in GPA.

\section{MATERIALS and METHODS}

After approval from Hacettepe University Vasculitis Center, a retrospective search was performed in their database to identify patients diagnosed with GPA. Hacettepe University Vasculitis Center has approval by the Institutional Review Board/Ethics Committee for retrospective studies. Ninety patients diagnosed with GPA between 1993 and 2018 were indentified. Further identification of patients having $\mathrm{CT}$ and/or MRI scans of ENT region was made by using Picture Archiving and Communication System and Hospital Information System in Hacettepe University Hospital. Inclusion criteria were as follows: 1-CT and/or MRI scans of ENT region performed due to high clinical suspicion of GPA at onset, 2- CT and/or MRI scans performed due to ENT related symptoms during follow up. The patients with tecniqually inadequate scans or having a history paranasal sinus surgery were excluded.

All CT scans were acquired with 16 slice CTs (Siemens, Erlangen, Germany; GE Healthcare, Milwaukee, Wisconsin). The examinations were performed in the supine position without gantry tilt. The scanner settings were adjusted to the range of the scanned area. The parameters of a standard protocol for CT scans are provided in table 1. The CT scans of paranasal sinuses and temporal bone were performed without intravenous contrast injection. The CT scans of the neck were acquired after IV injection of non-ionic contrast material with a delay of 35 seconds.

Axial images with bone and soft tissue windows, coronal reformatted images (thickness: 1-1.25 mm

Table 1. Parameters of a standard CT scan protocol

\begin{tabular}{|l|c|c|c|}
\hline \multicolumn{1}{|c|}{ Parameters } & & & \\
& Paranasal sinus CT & Nasopharynx/Neck CT & Temporal CT \\
\hline Slice thickness, mm & $1-1.25$ & $2.5-3$ & $1-1.25$ \\
\hline Reconstruction thickness, mm & $1-1.25$ & $1-1.25$ & $0.6-0.625$ \\
\hline Kilovoltage peak (kVp) & $100-120$ & 120 & $120-140$ \\
\hline Average effective miliampere-seconds (mAs) & $50-100$ & $95-120$ & $80-180$ \\
\hline Rotation time (seconds) & $0.5-0.7$ & $0.7-075$ & $0.75-1$ \\
\hline
\end{tabular}


All MR studies were performed at 1.5 T MR scanners (Philips Healthcare, Best, the Netherlands; GE Healthcare, Milwaukee, Wisconsin; and Siemens, Erlangen, Germany). A routine protocol for nasopharynx/neck MRI included coronal, sagittal and axial T1-weighted images, coronal short tau inversion recovery (STIR) image, axial T2-weighted images with fat saturation, post contrast axial and coronal T1-weighted images with fat saturation and diffusion weighted imaging ( $B=\max 800$ or 1000). Post contrast images were acquired after intravenous bolus injection of $\mathrm{Gd}$-based contrast material. The parameters of a routine protocol for nasopharynx/ neck MRI are provided in Table 2.

All CT and MRls were reviewed by a neuroradiologist (E.B.) with 5 year experience in head and neck radiology. The imaging features evaluated in $\mathrm{CT}$ scans were as follows; 1-opacifications/inflammatory signal changes in the nasal cavity and/or paranasal sinuses, 2- mucosal ulcerations and/or septations in the nasal cavity, 3-presence and location of partial or complete osseous erosions, septal perforation 4- presence and location of paranasal neo-osteogenesis, 5-periantral soft tissue infiltration, 6-opacifications/signal changes in the mastoid cells and/or tympanic cavity, 8- mastoid sclerosis, 9- nasopharyngeal thickening and/or ulceration, 10-parapharyngeal infiltration, 11-signal, enhancement and diffusion characteristics of nasopharyngeal involvement, 12-subglottic stenosis, 13-dural thickening and/or calcification.

Descriptive statistics were expressed as mean \pm $\mathrm{SD}$; categorical variables were presented as count and percentage.

Table 2. The parameters of a standard protocol for nasopharynx/neck MRI

\begin{tabular}{|l|c|c|c|c|}
\hline \multirow{2}{*}{ Sequence type } & T1- weighted SE & STIR & T2-weighted FSE & DWI \\
\hline FOV, cm & $23 \times 23-26 \times 26$ & $26 \times 26$ & $23 \times 23$ & $26 \times 22$ \\
\hline $\begin{array}{l}\text { Section thickness/ } \\
\text { spacing, mm }\end{array}$ & $4-4.2 / 5-5.4$ & $4.2 / 5.2$ & $5.4 / 4.2$ & $4.2 / 5.4$ \\
\hline TR/TI, milliseconds & $480-600$ & $6120 / 160$ & 6520 & $3450 / 165$ \\
\hline TE, milliseconds & $7-8$ & 35 & 85 & 76 \\
\hline Matrix size & $512 \times 512$ & $512 \times 512$ & $512 \times 512$ & $256 \times 256$ \\
\hline
\end{tabular}

FOV: Field of view; FSE: Fast spin echo; DWI: Diffusion weighted imaging; TE: Echo time; TI: Inversion time; TR: Repetition time; SE: Spin echo; STIR: Short tau inversion recovery

\section{RESULTS}

One patient with tecniqually inadequate paranasal sinus CT and one patient having a history of paranasal sinus surgery were excluded. A final number of 48 patients (mean age at imaging: $48.2 \pm 18$ years, F/M: 28/20) were included in the study.

Thirty-eight patients (79\%) had paranasal sinus CT, two of them also had MR scans. Thirty paranasal sinus CT (79\%) and one MR scan were performed due to high clinical suspicion of GPA, eight paranasal sinus CT (21\%) and one MR scan were performed due to a new ENT related symptom (nasal discharge, loss of smell exc.) during follow-up. A summary of imaging findings is provided in table 3.

Seventeen patients (35\%) were evaluated with nasopharynx/neck imaging; nine patients with MRI, 5 patients with $\mathrm{CT}$ and three patients with both $\mathrm{CT}$ and MRI. Patients had hoarseness, stridor, difficulty in breathing, fascial paralysis or ear pain prior to imaging. Four patients had suspicion of nasopharyngeal cancer.

Five patients were evaluated with temporal $\mathrm{CT}$; three patients at onset, two patients during follow up with the symptoms of hearing impairment \pm facial paralysis or otorrhea.

The erosive osseous changes were frequent in nasal septum either alone $(n=3)$ or with involvement of more lateral structures, i.e. turbinates, ethmoid lamella, medial maxillary wall $(n=8)$. Maxillary wall 


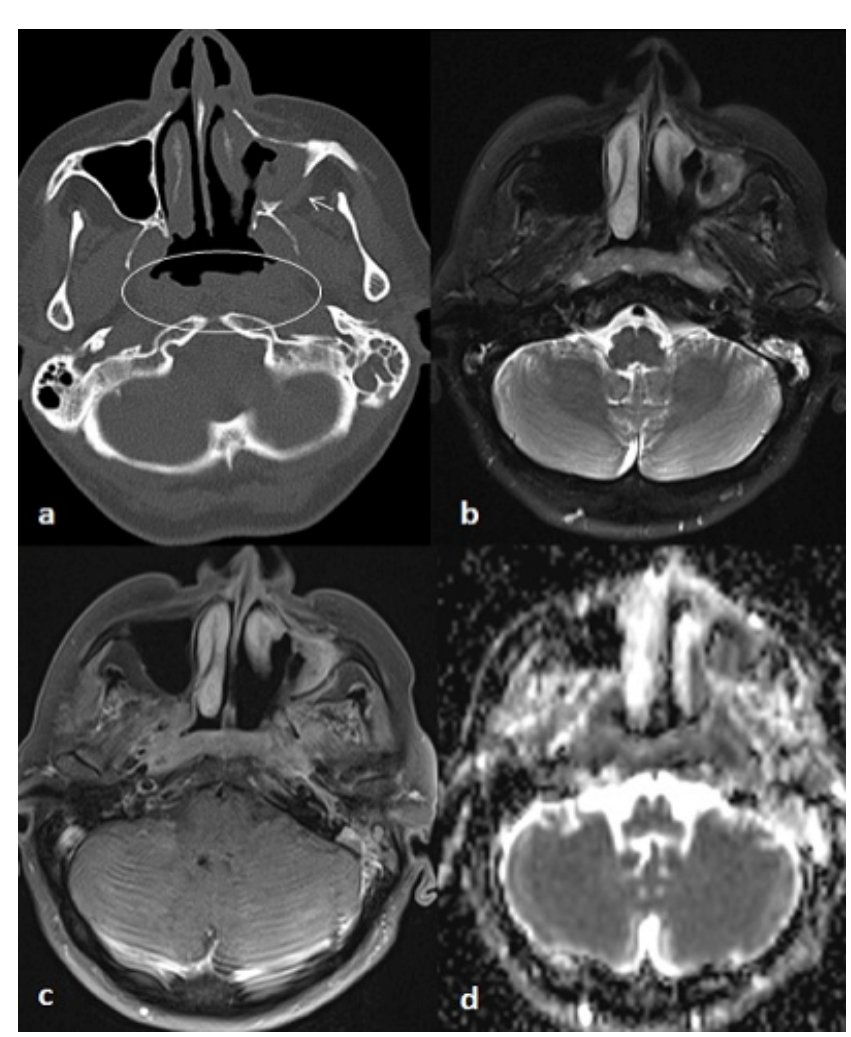

erosion without involvement of mid-line structures was detected in six patients (Figure 1a).

Septal perforation was detected in three patients; in one patient at disease onset, in two patients during follow-up.

Neo-osteogenesis invariably affected maxillary sinuses either unilaterally $(n=5)$ or bilaterally $(n=4)$ (Figure 2). One patient with bilateral maxillary involvement also had neo-osteogenesis of unilateral frontal sinus; one patient had extensive neo-osteogenesis with involvement of all paranasal sinuses except for the left frontal sinus. Neo-osteogenesis was detected in follow-up studies in six patients (66\%). Six patients with neoosteogenesis (66\%) had also accompanying erosive/destructive bony changes. MRI was performed only in four patients with nasopharyngeal thickening. Affected areas of nasopharynx showed low T2 signal $(n=4)$, diffuse $(n=2)$ or heterogeneous $(n=2)$ contrast enhancement and restricted diffusion (mean $A D C$ value=0.582) (Figure $1, \mathrm{~b}-\mathrm{d})$.

Figure 1. Nasopharynx CT and MRI of a 47 year old female GPA patient with symptoms of rhinosinusitis and impaired hearing. CT image with bone window (a) shows opacifications in left maxillary sinus with bone destruction and retroantral soft tissue infiltration (arrow). Also note thickening of nasopharyngeal wall with surface irregularities (circle). T2 weighted (b), postcontrast T1 weighted (c) and ADC map images (d) demonstrate imaging characteristics of granulomatous inflammation in both maxillary sinus and nasopharynx; low T2 signal intensity, contrast enhancement and restricted diffusion with low ADC values, respectively.

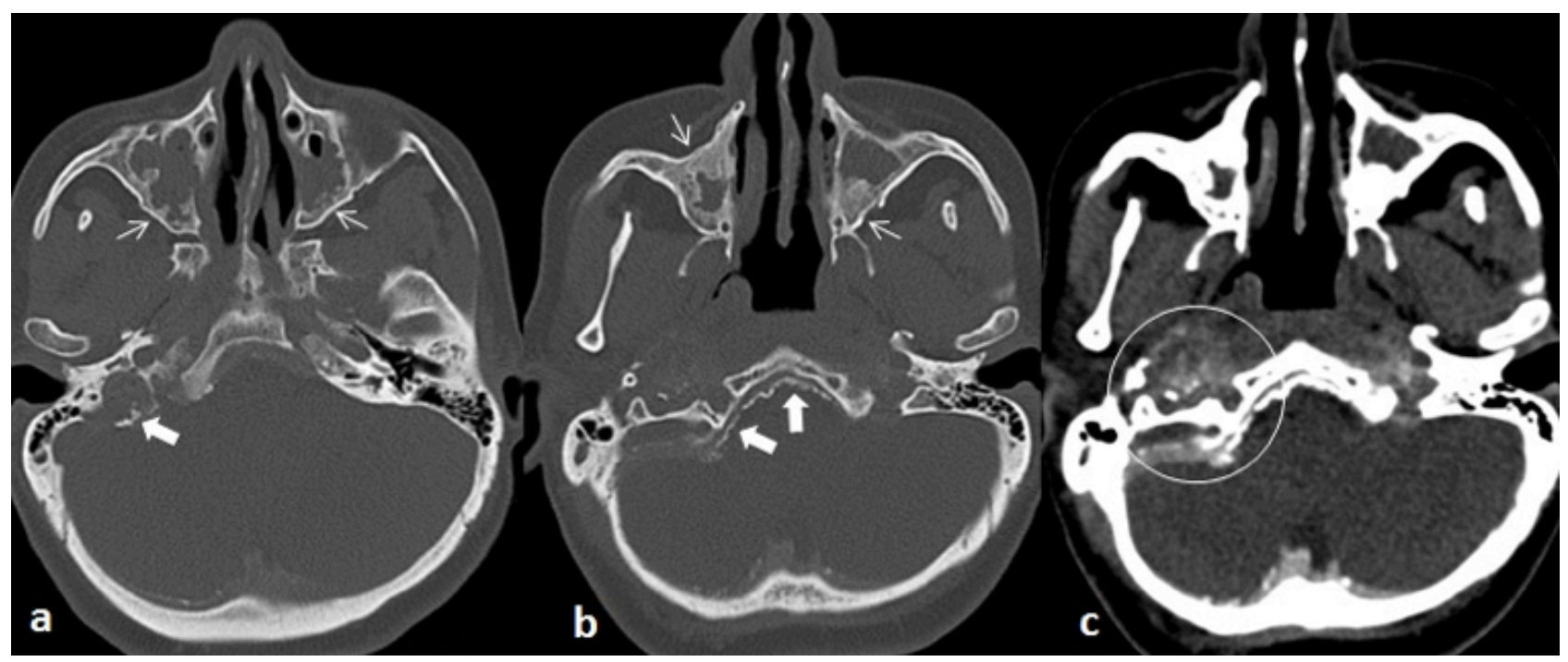

Figure 2. Nasopharynx CT of a 50 year old GPA patient presenting with hearing loss in her right ear. Bone window images (a, b) show neoosteogenesis and soft tissue opacifications in both maxillary sinuses (thin arrows). Also note dural calcifications in posterior fossa (thick arrows). Soft tissue window image (c) better delineates dural thickening and contrast-enhanced, calcified soft tissue thickening in bilateral carotid space (circled in right side). 
Table 3. Summary of imaging findings

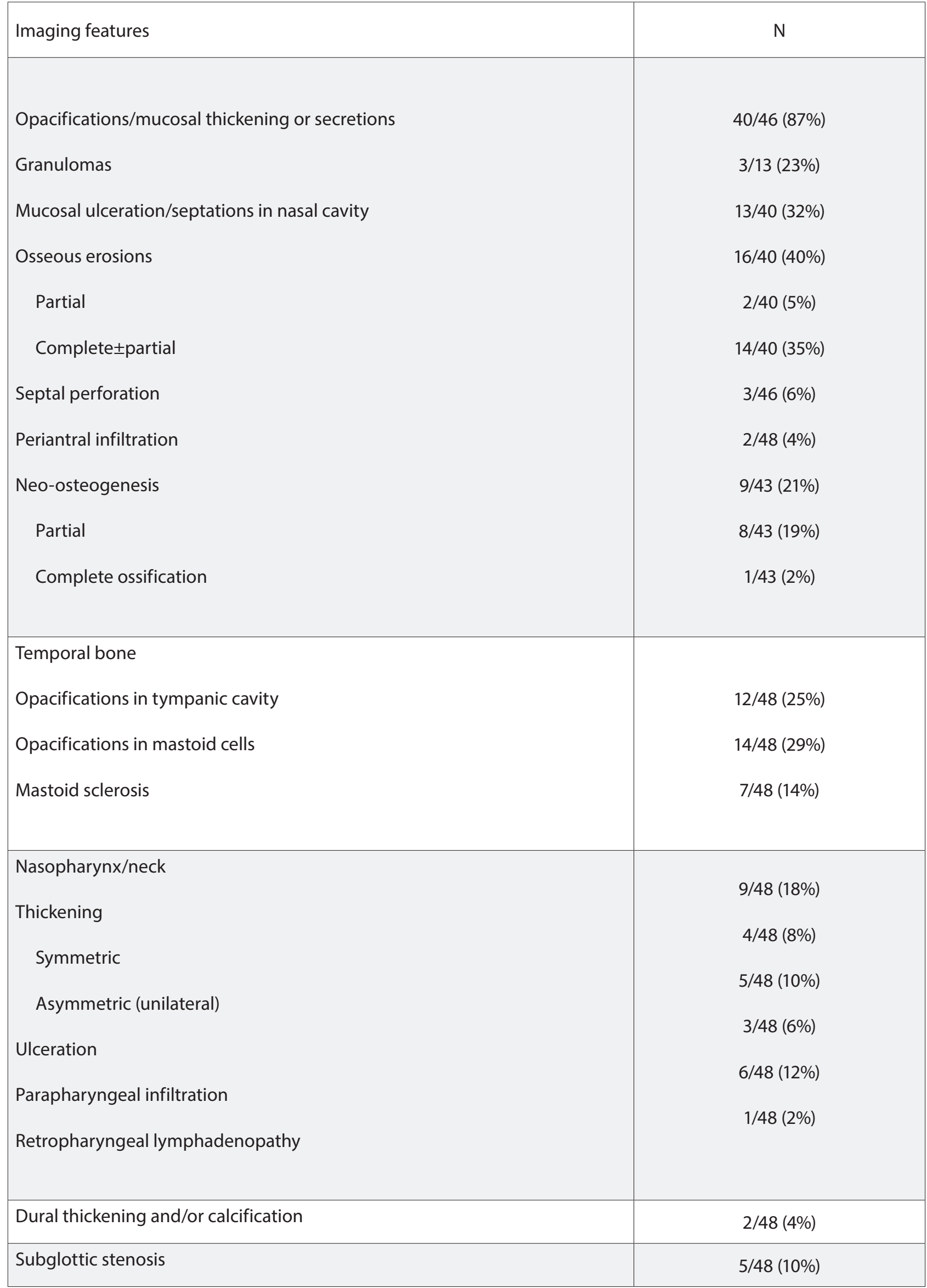


Early treatment with effective agents enables to decrease morbidity in GPA, which underlines the role of radiologist in suggesting the diagnosis of GPA in clinically inconclusive cases. Most of the imaging features of GPA overlap with those of other diseases; therefore familiarity with more specific finding is important to suspect the diagnosis. In this relatively large cohort, we aimed to reveal frequencies of imaging findings previously defined as suggestive for GPA and to investigate the radiologic findings of previously under-recognized nasopharyngeal involvement.

Paranasal sinus opacifications in CT scans are highly non-specific and could represent sinusitis of any origin, retention cyst or polyposis. Lohrmann et al. found that mucosal thickening is a frequent finding in both nasal cavity and paranasal sinuses with the ratios of $61 \%$ and $75 \%$, respectively [11]. They did not specify the imaging characteristics of mucosal thickening in nasal cavity. Benoudiba et al. found that nodular mucosal thickening could favor the diagnosis of GPA [6]. Thirteen (32\%) patients in our cohort showed irregular mucosal thickening with ulcerations and septations in nasal cavity. It probably reflects the histopathology of GPA, and this finding could favor the diagnosis especially when coexistent with bony changes. Paranasal sinus opacifications were frequent similar to previous studies. Associated MRI findings that reflect the granulomatous nature of the opacifications such as low T2 signal intensity or restricted diffusion could favor the diagnosis in clinically suspected cases. In our series, eight patients with sinonasal opacifications had also concurrent MR studies and only $3(37 \%)$ of them showed typical signal and diffusion characteristic of granulomas.

Bone destruction at the mid-line structures and periantral soft tissue infiltration associated with bone demineralization were reported as suspicious findings for GPA [6]. Bone erosions/destruction results from avascular necrosis due to fibrinoid necrosis of the small to medium arteries accompanied by granulomatous inflammation [12]. The frequency of partial or complete osseous erosions (40\%) in our study were slightly lower than the frequencies of bony destruction in nasal cavity (57\%) and paranasal sinuses (54\%) reported by Lohrmann et al and the frequency of bony erosions (62\%) reported by Grindler et al $[7,11]$. Erosive/destructive bony changes were described to initiate at the mid-line structures and 14 proceed laterally to involve turbinates, medial maxillary walls and other sinuses in previous reports [6]. Our results were similar; most of the cases in our series $(n=11)$ demonstrate the same pattern, although there were cases $(n=8)$ with erosions/destructions in maxillary sinus wall sparing the midline. Especially if associated with periantral soft tissue infiltration, isolated sinus wall destruction could be challenging to differentiate from invasive fungal infections or malignancies. We had two cases with periantral soft tissue infiltration; one with associated maxillary wall destruction and all two with associated neo-osteogenesis of the sinus wall.

Septal perforation should suggest the diagnosis in suspected cases of GPA. Contrary to the study of Grindler et al. in which septal perforation was not an infrequent finding (35.1\%), it is infrequently (6\%) detected in our study. This could be due the differences in the study designs; most of the examinations included in our study were performed at disease onset, so cases with the findings of a longstanding disease were mostly precluded.

Neoosteogenesis is a new bone formation inside the affected sinus wall. It has a distinctive appearance on $\mathrm{CT}$ with an irregular line of corticated bone inside, separated from the normal wall by an area of less dense bone [12]. It particularly affects maxillary sinuses $[7,12]$. The process of neoosteogenesis is reported to be seen in up to $50 \%-78 \%$ of patients $[7,12]$. Similarly, maxillary sinus involvement was invariable in our patients with neo-osteogenesis, although the finding was not as frequent (21\%) as the previous reports. This again probably reflects the fact that most of the imaging examinations evaluated in our study was acquired at disease onset; therefore a chronic process like neo-osteogenesis could be missed. Accordingly, neo-osteogenesis was more frequently detected in follow-up scans (66\%). Therefore, it could be suggested that it is more likely to find osseous erosions-destructions than neo-osteogenesis in a CT of a patient who has not been diagnosed yet.

Co-existence of bony destruction and neo-osteogenesis was found to be highly suggestive of GPA in patients presenting with symptoms of chronic rhinosinusitis [5]. Concordantly, most of the patients with neoosteogenesis had also accompanying erosive/destructive bony changes in our study.

The laryngeal and endobronchial involvement may present radiologically with circumferential mucosal 
thickening, irregularity and ulceration [5]. Subglottic stenosis is defined as the most frequent airway manifestation and caused by chronic mucosal inflammation with eventual fibrosis. Airway involvement is typically detected as a late manifestation of GPA, although an isolated form could manifest with undetectable ANCA levels which makes the diagnosis challenging. The reported frequencies of subglottic stenosis are variable and ranges from $7 \%$ to $50 \%$ [3, $7,13]$. Subglottic stenosis was not a common finding (10\%) in our study; detected in examinations of five patients and three of them manifested late during the course of the disease (Figure 3).

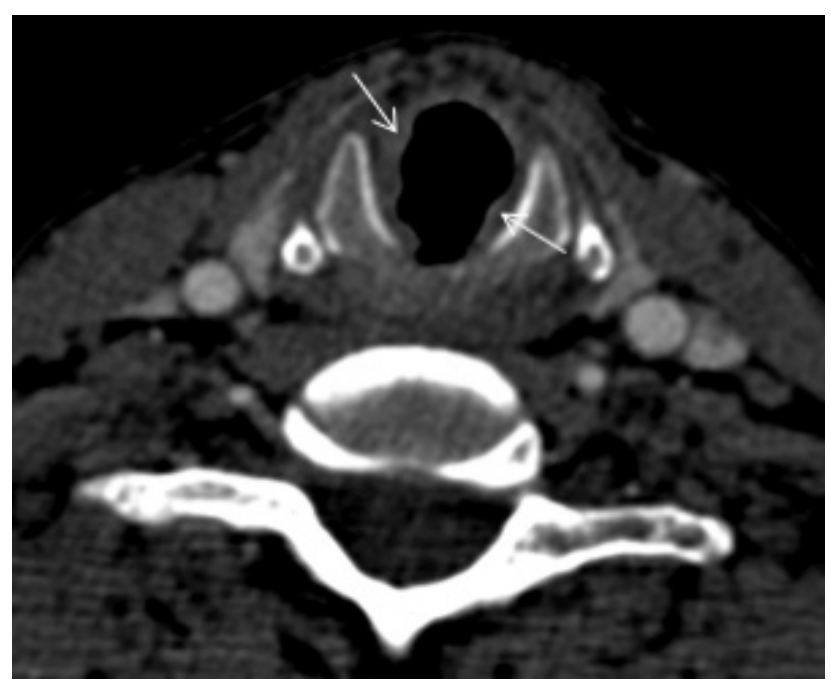

Figure 3. Nasopharynx CT of a 44 year old male patient presenting with hoarseness. Axial image shows irregular, circumferential soft tissue thickening at subglottic region (arrows).

Otologic manifestations are seen in up to $40 \%$ of patients with GPA [5]. The most frequent clinical manifestation is conductive hear loss due to serous otitis media. It is caused by obstruction of the eustachian tube due to direct extension of adjacent sinonasal disease. CT manifestations are non-specific and consist of opacifications of the middle ears and mastoid cells with thickening of bony septa. Our findings were similar, and most of the patients with temporal involvement (70\%) had coexistent sinonasal and/or nasopharyngeal involvement. We did not detect a distinctive sing of a granulomatous inflammation with bone destruction mimicking coalescent otomastoiditis.

Nasopharyngeal manifestations were under-recognized radiologically in previous studies; imaging findings were defined only in few case reports. Nasopharyngeal involvement was not infrequent (23\%) in our cohort with the findings of Hacettepe University Vasculitis Center. symmetric or asymmetric wall thickening with/ without parapharyngeal infiltration, mucosal ulceration and retropharyngeal lymphadenopathy. Nasopharyngeal involvement was detected particularly in the scans at onset; involvement during disease follow-up was seen only in one patient. The limited data in literature comes from CT or conventional MRI characteristics of nasopharynx and/ or eustachian tube involvement without specifying diffusion properties of inflammatory infiltration [8-10]. In our series, MRI characteristics were similar with the other ENT parts of involvement and reflected the granulomatous nature of the inflammation. Diffusion restriction was inevitable in affected parts of nasopharynx, further complicating the differentiation of GPA from nasopharyngeal cancer, lymphoma or from the other granulomatous inflammatory diseases. Overlapping clinical and imaging manifestations in nasopharyngeal involvement could cause diagnostic and eventually treatment delays. This underlines the role of any potential imaging finding that could discriminate nasopharyngeal involvement of GPA from other overlapping disease entities. Therefore, a detailed search for discriminative MRI findings including diffusion characteristics with future comparative studies is strongly recommended.

Our study has some limitations due to its retrospective design. CT and MR examinations were performed with different scanners and with different parameters, which could affect the image evaluation. Additionally, not all or the abnormal findings detected in CT were further characterized with MRI.

\section{CONQUSION}

Sinonasal osseous erosions/destruction is a relatively common and highly suggestive finding especially when co-existent with mucosal ulcerations/irregularities in nasal cavity, neoosteogenesis and nasopharyngeal thickening in a suspected case of GPA. Nasopharyngeal involvement is not infrequent and could overlap with other entities such as nasopharyngeal cancer or lymphoma. Future comparative studies to identify imaging discriminators between these disease entities are needed.

\section{ACKNOWLEDGEMENT}

This retrospective research was performed using

(1)


[1] Jennette JC, Falk RJ, Andrassy K, et al. Nomenclature of systemic vasculitides. Proposal of an international consensus conference. Arthritis Rheum 1994; 37: 187-92.

[2] Comarmond C, Cacoub P. Granulomatosis with polyangiitis (Wegener): clinical aspects and treatment. Autoimmun Rev 2014; 13: 1121-5.

[3] Kuhn D, Hospowsky C, Both M, et al. Manifestation of granulomatosis with polyangiitis in head and neck. Clin Exp Rheumatol 2018; 36 Suppl 111: 78-84.

[4] Borner U, Landis BN, Banz Y, et al. Diagnostic value of biopsies in identifying cytoplasmic antineutrophil cytoplasmic antibody-negative localized Wegener's granulomatosis presenting primarily with sinonasal disease. Am J Rhinol Allergy 2012; 26: 475-80.

[5] Pakalniskis MG, Berg AD, Policeni BA, et al. The Many Faces of Granulomatosis With Polyangiitis: A Review of the Head and Neck Imaging Manifestations. AJR Am J Roentgenol.2015; 205: W619-29.

[6] Benoudiba F, Marsot-Dupuch K, Rabia MH, et al. Sinonasal Wegener's granulomatosis: CT characteristics. Neuroradiology 2003; 45: 95-99.

[7] Grindler D, Cannady S, Batra PS. Computed tomography findings in sinonasal Wegener's granulomatosis. Am J
Rhinol Allergy 2009; 23: 497-501.

[8] Helmberger RC, Mancuso AA. Wegener granulomatosis of the eustachian tube and skull base mimicking a malignant tumor. AJNR 1996; 17: 1785-90.

[9] La Rosa C, Emmanuele C, Tranchina MG, et al. Diagnostic consideration for sinonasal Wegener's granulomatosisclinically mistaken for carcinoma. Case Rep Otolaryngol.2013; 2013: 839451.

[10] Mohapatra A, Holekamp TF, Diaz JA, et al. Atlantoaxial subluxation and nasopharyngeal necrosis complicating suspected granulomatosis with polyangiitis. J Clin Rheumatol 2015; 21: 156-9.

[11] Lohrmann C, Uhl M, Warnatz K, et al. Sinonasal computed tomography in patients with Wegener's granulomatosis. J Comput Assist Tomogr. 2006; 30: 122-5.

[12] Lloyd G, Lund VJ, Beale T, et al. Rhinologic changes in Wegener's granulomatosis. J Laryngol Otol 2002; 116: 565-69.

[13] Polychronopoulos VS, Prakash UB, Golbin JM, et al. Airway involvement in Wegener's granulomatosis. Rheum Dis Clin North Am 2007; 33: 755-75 